\title{
Developing Architectural Lighting Designs to Improve Sleep in Older Adults
}

\author{
Mariana G. Figueiro*, ${ }^{*}$, Elyse Saldo ${ }^{2}$, Mary S. Rea ${ }^{2}$, Karen Kubarek ${ }^{2}$, Julie Cunningham ${ }^{3}$ and Mark \\ S. Rea ${ }^{1}$ \\ ${ }^{1}$ Lighting Research Center, Rensselaer Polytechnic Institute, Troy, NY, USA \\ ${ }^{2}$ Department of Biology, The Sage Colleges, Troy, NY, USA \\ ${ }^{3}$ Department of Psychology, The Sage Colleges, Troy, NY, USA
}

\begin{abstract}
This paper discusses a proposed 24-hr lighting scheme for older adults that can positively impact the aging visual, circadian and perceptual systems. New lighting was installed in eight private rooms in an assisted living facility. Measurements of residents' sleep quality and circadian rest-activity patterns were obtained, before and after the new lighting was installed. Consistent with predictions based upon previous research, the subjects who completed the study showed an improvement in sleep quality and rest/activity rhythms under the new 24-hr lighting scheme. In addition, all study participants reported a strong preference for the 24-hr lighting. The new lighting not only provides older adults with good lighting for performing their routine visual tasks, but also promotes high circadian light stimulation during the day and low circadian light stimulation at night. Although not studied here, but also discussed as part of the 24-hr lighting scheme, is the impact of a previously studied, novel night-light system that provides older adults with enhanced perceptual cues for nighttime navigation within the room. The new 24-hr lighting scheme appears to have important practical implications for improving the quality of life for seniors and will hopefully be adopted by architects, lighting specifiers and engineers.
\end{abstract}

Keywords: Senior housing, lighting, visual system, circadian system, sleep quality.

\section{INTRODUCTION}

Light reaching the retina allows us to see [1] and it synchronizes our circadian rhythms to the 24-hr solar day [2]. Lighting also affects the perceptual system enabling us to orient ourselves to the spatial environment and to help maintain postural control and stability [3]. There are two major problems with residents in all senior health care facilities: poor sleep quality and falls. New approaches to lighting design can be used to address these two major problems. This paper discusses an integrated, 24-hr lighting design strategy for older adults that takes into account the needs of the aging visual, circadian and perceptual systems [4]. Original results are presented from a demonstration project designed to test the effectiveness of light in improving sleep quality in older adults. A recent study of a novel night-light system that demonstrably improved postural stability in older adults is also summarized [5]. It is the authors' hope that a deeper understanding of the physiological changes to the aging visual, circadian and perceptual systems and how lighting affects these systems will result in wider use of light as a nonpharmacological tool to help improve the quality of life of older adults.

\section{BACKGROUND}

\section{The Human Visual System}

The eye consists of optical and neural parts. The function of the optical parts of the eye is to place a focused, clear im-

*Address correspondence to this author at the Lighting Research Center, Rensselaer Polytechnic Institute, Troy, NY, USA; E-mail: figuem@rpi.edu age of the outside world on the retina. The optical parts of the eye assure that light is transmitted through the eye with minimal absorption and scattering. The function of the retina is to absorb light signals and convert them to electrical signals. The human eye has photoreceptors that convert radiant energy into neural signals for processing by the brain, a phenomenon called phototransduction. Until recently, four types of photoreceptors had been identified: rods and short, middle, and long wavelength cones. Rods allow us to see at night and cones allow us to see during the day, discriminate details and see colors. The human visual system can process information over a large range of luminances (about $12 \mathrm{log}$ units). In order to cope with a wide range of retinal illuminances from nighttime light levels $\left(0.000001 \mathrm{~cd} / \mathrm{m}^{2}\right)$ to outdoor light levels $\left(100,000 \mathrm{~cd} / \mathrm{m}^{2}\right)$, the human visual system changes its sensitivity continuously, a process called adaptation. As confirmed by many studies (reviewed in [1]), at typical office illuminance levels (500 lux on the task surface or approximately 100 lux at the eye), visual performance is near maximum for young adults (for targets of high contrast and large size). The process of adaptation also influences the spectral sensitivity of the visual system because different combinations of photoreceptors are operating at different retinal illuminances. At photopic light levels (adaptation luminances higher than approximately $1 \mathrm{~cd} / \mathrm{m}^{2}$ ), typical of building interiors, retinal response is dominated by cone photoreceptors in the fovea and periphery. The luminous efficiency function for the CIE Standard Photopic Observer has a maximum sensitivity at $555 \mathrm{~nm}$. At scotopic light levels, (adaptation luminances below $0.001 \mathrm{~cd} / \mathrm{m}^{2}$ ) only rod photoreceptors respond to light stimulus and the fovea is not 
operating. The luminous efficiency function for the CIE Standard Scotopic Observer has a maximum sensitivity at $507 \mathrm{~nm}[6,7]$. At mesopic light levels, (adaptation luminances between approximately 0.001 and $\left.1 \mathrm{~cd} / \mathrm{m}^{2}\right)$, both rods and cone photoreceptors are active. There is no official luminous efficiency function for the Standard Mesopic Observer. Spatial distribution of light on the retina is critical to vision. Through optical refraction by the cornea and lens in the eye and by neural-optical enhancements by the retina, the spatial distribution of objects and textures in the environment can be processed with high fidelity by the visual system. The visual system can fully process light pulses as short as 80 milliseconds, so the temporal characteristics of light are generally not considered in the lighting design process for the visual system [1].

\section{The Human Circadian System}

The world rotates around its axis and as a result, all creatures exposed to daylight on earth experience 24-hour cycles of light and dark. Biological rhythms are self-sustaining oscillations with a set of species-specific characteristics, including amplitude, phase, and period [8]. Living organisms have adapted to this daily rotation of the earth by developing biological rhythms that repeat at approximately 24 hours. These are called circadian rhythms (Latin: circa, about; dies, a day). Circadian rhythms are generated endogenously (internal to the body) and are constantly aligned with the environment by zeitgebers, factors exogenous or external to the body. In mammals, circadian rhythms are regulated by an internal biological clock (pacemaker) located in the suprachiasmatic nuclei (SCN) of the hypothalamus of the brain [8]. The SCN is a self-sustaining oscillator that maintains its daily activities for weeks when isolated and cultured. The SCN in humans has a natural period that is slightly greater than 24 hours and environmental cues can reset and synchronize the SCN daily, ensuring that the organism's behavioral and physiological rhythms are in synchrony with the daily rhythms in its environment. The light/dark cycle is the main synchronizer of the SCN to the solar day [9] and reaches the SCN via the retinohypothalamic tract (RHT). In 2002, the intrinsically photosensitive retinal ganglion cell (ipRGCs), a novel photoreceptor type in the retina was discovered [10]. The ipRGCs are central to the "non visual" responses to light by the retina, most notably the regulation of circadian rhythms. It has been suggested that a combination of classical photoreceptors and the ipRGCs participate in how the retina converts light signals into neural signals for the circadian system $[11,12]$. The exact amount of light needed to activate the circadian system is still under debate, especially outside laboratory conditions. Based on studies that have been conducted under controlled conditions, it is now accepted that light levels needed to activate the circadian system are higher than those needed to activate the visual system, so one can see well in the environment, but may not be getting enough light to stimulate the circadian system. The circadian system is a "blue-sky detector," maximally sensitive to wavelengths shorter than $470 \mathrm{~nm}$ [1214]. Unlike the visual system, the circadian system simply detects changes in overall irradiance, that is, the circadian system does not care about pattern information or image forming [15]. Perhaps the largest difference between the visual and circadian systems is associated with when light is registered on the retina $[15,16]$. Light can either advance or delay the SCN, that is, light applied after wake-up time will cause one to go to bed earlier and wake up earlier while light applied before bed times will cause one to go to bed later and wake up later [16]. The circadian system takes longer to respond than the visual system. It takes approximately 5-10 minutes for a measurable effect of light on markers of the circadian system to be seen [17]. Finally, the circadian system is concerned with changes in light levels, rather than absolute light levels [18]. For example, an older person who stays in a dim room all day long will suppress more melatonin when exposed to a given light at night than would a farmer, who has experienced very high outdoor light levels. Contrast in light exposure during the day and night is important to maintain a healthy and synchronized circadian system.

\section{The Human Perceptual System}

There are many theoretical models of how people perceive the three-dimensional world around them. Previc [19] describes a comprehensive model that incorporates all of these models into one. This model consists of four behavioral realms: peripersonal (visuomotor operations in nearbody space), focal extrapersonal space (visual search and object recognition), action extrapersonal (orienting in a topographically defined space), and ambient extrapersonal (orienting in earth-fixed space). All of these areas together combine for a total 3-D perception of space; however, the ambient extrapersonal realm is the area that is most important for the perception of surface planes and orienting one's self to these planes. Its three main functions are spatial orientation, postural control, and locomotion. All of these functions are necessary to navigate changes in the world such as inclines, obstacles, and edges.

Gibson [3] describes the senses as perceptual systems. There are five main perceptual systems: the orienting system, the auditory system, the haptic (cutaneous or touch) system, the taste-smell system, and the visual system. These systems use information gathered from the environment to enable humans to perform activities such as spatial orientation, postural control, and locomotion. The sensory systems involved with the ambient extrapersonal realm are visual perception (ambient motion, slant), vestibular, and somatosensory/proprioceptive (orientation). However, the visual system is the dominant system involved. The environment surrounding an individual is the source of all stimulation for perception. A person gains perspective by the many intersecting planes that make up the environment. When these planes are illuminated, they reflect light and project structure. The different ways these planes are connected offer further information about the environment. For example, one plane in front of another represents an edge, while two plane surfaces joining one another represents a corner. Horizontal cues are obtained both from visual sensations of horizontality and by vestibular feedback cues. Architectural features can be used to provide information about the environment and therefore improve navigation in the space. Light is a stimulus to the visual system and visual information provides a spatial reference for self-position and location of obstacles within a person's surroundings. Removal of visual cues by closing the eyes has been shown to result in increased body sway. Therefore, lighting schemes that enhance architectural 
features should be used to help with navigation and orientation in a space.

\section{Changes to the Aging Visual System}

Although visual capabilities decline from the age of 20 years, the human visual system can often be considered "young" until about the age of 40 years [20]. Then, changes to the aging eye become more noticeable as visual capabilities decrease. Trouble focusing on objects at different distances (known as presbyopia) is particularly common after age 45 . Hardening of the crystalline lens capsule and, perhaps, atrophy of the ciliary muscles are the primary causes of lost accommodation. By age 65, variable accommodation is nearly impossible and multi-focal lenses are required. As a person grows older, less light reaches the back of the eyes because the pupil gets smaller and the crystalline lens inside the eye becomes thicker, absorbing more light. A 60-year old receives about $1 / 3$ of as much light at the retina as a 20 -year old. The lens also begins to scatter more light as one ages, adding a "luminous veil" over images on the retina, which reduces the distinctness (or contrast) of objects and the vividness of colors. Reds begin to look like pinks, for example. Moreover, the older eye loses some sensitivity to shortwavelengths (blue light) due to progressive yellowing of the crystalline lens. Because of neural changes that occur to the aging eye, older adults take longer to adapt to changes in brightness (e.g., transitioning from indoors to outdoors). Last, but not least, chances of having age-related eye diseases (cataract, glaucoma, macular degeneration, and diabetic retinopathy) are greater after age 60 to 65 years. Because of these changes to the aging eye, more light on the task is required for older adults to perform visual tasks, especially those of low contrast and small size. The aging visual system is also more sensitive to glare, so lighting that meets the need of the aging eye should be glare free (i.e., no direct or reflected view of the light source is acceptable). Because the older adult's visual system cannot completely adapt to dim conditions, light levels in transitional spaces (such as hallways and entrance foyers) should be balanced with those of the adjacent spaces. Intermediate light levels in transitional spaces that lead from bright to dim areas should be created [21]. This will enable older adults to adapt more completely as they move through the different spaces. Reflecting light off of light color surfaces will reduce the likelihood that pools of light and dark are formed in the space while increasing light uniformity.

\section{Changes to the Aging Circadian System}

Sleep disturbances increase as we age and disruption of the circadian system is one important reason. Surveys indicate that 40 to 70 percent of the oldest members of the population (over 65 years old) suffer from chronic sleep disturbances [22]. In general, this group of adults tends to go to bed earlier in the evening and wake earlier in the morning than younger adults. Frequent nocturnal awakenings, difficulty falling asleep, and an increased number of naps during the day are also more common in the oldest adults. Sleep disturbances are associated with decreased physical health, including increased cardiovascular problems, disruption of endocrine functions, and decline of immune functions [23].

Studies of the circadian pacemaker, the central component of the circadian system, have shown reduced neuronal activity in the SCN of the elderly, especially after the age of 80 [24], and a reduced circadian rhythm amplitude after the age of 50 [25]. This suggests that, at a molecular level, the SCN becomes less responsive to entrainment stimuli such as light-induced neural signals from the retina. Further, it is hypothesized that some of the neural processes involved in entrainment might be dysfunctional or less effective as we age [26]. Light information travels from the retina to the SCN through the RHT. Disturbances in circadian rhythms leading to poor sleep in older adults can be the result of dysfunctional circadian pathways or a pathway that cannot process light information with as much fidelity. Also, the first stage of phototransduction (when light signals are converted into neural signals) is negatively affected: older adults not only have reduced optical transmission at short wavelengths, which is maximally effective for the circadian system, they also lead a more sedentary indoor lifestyle, with less access to bright light during the day. In fact, research has demonstrated that middle-aged adults are exposed to approximately 58 minutes of bright light per day [27] while older adults in assisted living facilities were exposed to bright light for only 35 minutes a day [28]. Moreover, adults in nursing homes see as little as 2 minutes a day [29]. Finally changes in the amplitude and timing of melatonin and core body temperature rhythms may occur in older adults. Lower amplitude of melatonin rhythms may be associated with reduced sleep efficiency and deterioration of internal circadian rhythms, affecting hormone production, alertness, and performance [30]. Furthermore, earlier timing of melatonin rhythms peaks may induce earlier drops in core body temperature. Waking typically occurs about two hours after the minimum core body temperature, so the early waking times of older adults may be a result of earlier core body temperature troughs (reviewed in [31]. In addition, the time interval between the core body temperature minimum and waking time is reduced in elderly subjects [32].

\section{Changes to the Aging Perceptual System}

In addition to reductions in retinal illumination due to the aging eye, the quality of the retinal image is also compromised in older adults, possibly contributing to falls. Agedependent changes in the crystalline lens lead, in particular, to poorer spatial vision. As the crystalline lens ages it becomes less clear and scattered light reduces spatial resolution, not only reducing visual acuity but also elevating contrast threshold for all spatial frequencies [33]. Under low light levels even high contrast objects, such as door frames and objects on the floor, can go undetected, leading to disorientation and falls [34].

Neural changes also become problematic for the oldest people in our population. Gibson [3] describes a number of higher-order perceptual phenomena that can be compromised in seniors. In particular, an inability to orient oneself with respect to the environment becomes more common with aging. This neural weakness is confounded and amplified by optical changes in the eye (reduced retinal illuminance and spatial resolution) and, together, may contribute significantly to the increased incidence of falls found in older people.

\section{Previous Studies}

It is well established that lighting that is designed with the aging visual system in mind can help increase independ- 
ence in older adults. The Lighting Research Center (LRC) at Rensselaer Polytechnic Institute developed and published guidelines for designing lighting for older adults sponsored by AARP Andrus Foundation. The Illuminating Engineering Society of North America (IESNA) published a Recommended Practice with recommendations for lighting for older adults that meet the needs of the aging eye.

A new area of investigation is the impact of light on sleep efficiency of older adults. Studies using bright white light have demonstrated that light treatment can help reduce the negative impact of aging on circadian rhythms of sleep and wake, and thus, improve the quality and quantity of sleep in older adults, including those with Alzheimer's disease (AD). Bright white light exposure in the morning improved sleep in institutionalized older adults. Fetveit and colleagues [35] demonstrated that exposure to 2 hours of bright light in the morning for at least 2 weeks substantially improved sleep efficiency of older adults living in nursing homes. Alessi et al. [36] showed that five consecutive days of 30-minute exposure to sunlight, increased physical activity, structured bedtime, and control of light and noise at night resulted in a significant decrease in daytime sleeping in intervention participants compared to controls. Further, they showed that intervention participants had increased participation in social and physical activities as well as social conversation. Murphy and Campbell [37] have shown that light exposure in the evening can delay the circadian clock and help older adults sleep better at night and be more awake during the day. Fontana Gasio et al. [38] investigated the effectiveness of a 3 -week exposure to a low intensity (approximately 200 lux at the eye level) dawn-dusk simulation in improving disturbances of circadian rest-activity cycle, nocturnal sleep and/or cognitive functions in 13 demented patients. There were no significant changes in cognition, nor there was a modification of circadian rest-activity cycle. However, the main sleep episode was $1: 14 \mathrm{~h}$ earlier during treatment compared with before and after dusk-dawn simulation. Further, patients who underwent treatment tended to have shortened sleep latency, longer sleep duration, and less nocturnal activity than the control group. In parallel, nighttime light exposure tended to be reduced.

Studies have also demonstrated that bright white light exposure during the morning improves nighttime sleep, increases daytime wakefulness, reduces evening agitation behavior, and/or synchronizes rest/activity patterns of people with AD. These studies have used bright white light (at least 2500 lux and as high as 8000 lux) for at least 1 hour in the morning, and treatment was carried on for at least 2 weeks. Results showed greater sleep efficiency at night, decreased sleep during daytime hours and, in some cases, reduced agitation behavior [39-44]. Unattended exposure to bright white light during the entire day improved rest/activity rhythms of people with AD. Van Someren and colleagues [45] demonstrated that exposing 22 dementia patients to continuous bright indirect light (average of 1136 lux) over 4 weeks consolidated rest/activity rhythms of people with AD. Bright white light exposure in the evening has also been shown to improve quality of sleep and/or decrease nocturnal activity and severity of sundowning (evening agitation). Satlin and colleagues [46] demonstrated that evening exposure to bright light (1500 to $2000 \mathrm{lux}$ ) for 2 hours decreased nocturnal activity and severity of sundowning of $\mathrm{AD}$ patients. Figueiro and colleagues have conducted two studies showing the impact of blue light from light emitting diodes (LEDs) on sleep quality in elderly persons with varying AD symptoms [47, 48]. The first six-week study was conducted in February and March 2002 in a senior health care facility in Clifton Park, NY. During the evening from 18:00 to 20:00, four AD patients were exposed to light from tabletop light fixtures $(\sim 30$ $1 \mathrm{x}$ at the cornea of red LEDs) for 10 days. The red light exposure (RLE) condition was introduced as a control, because red light at this intensity should not activate the circadian system $[12,13,49]$. RLE was followed by 10 days of $\sim 301 \mathrm{x}$ of blue light exposure (BLE) using a similar fixture. Blue light was expected to activate the circadian system. All indi-

a

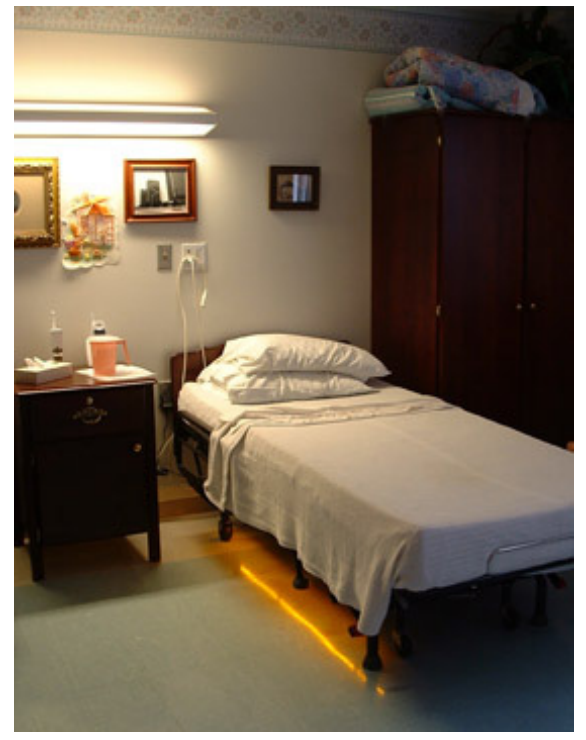

b

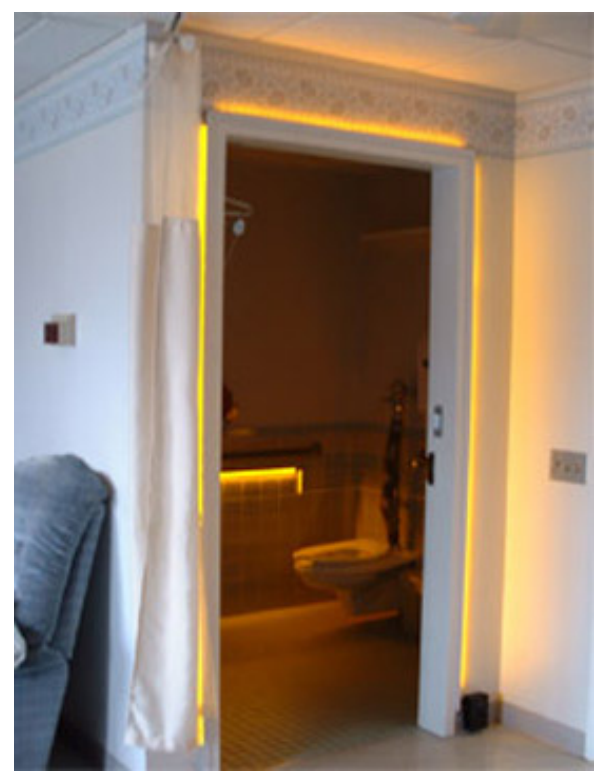

Fig. (1). a). Motion-sensor controlled amber LEDs installed under bed.* b). Motion-sensor controlled amber LEDs installed around doorway.*

* Notes: 1) photosensors were disconnected for the photos; 2) overhead fluorescent luminaire was kept off after installation of night-lighting. 
cators obtained in the study following BLE were consistent with theoretical expectations. The percentages of sleep revealed a statistically significant time by lighting conditions interaction $(\mathrm{p}=0.003)$. The percentages of time that subjects were asleep at 02:00 and at 04:00 were significantly greater $(\mathrm{p}=0.046$ and $\mathrm{p}=0.013$, respectively) after BLE than after RLE. Wrist-worn actigraphic recordings for two subjects found a greater consolidation of activity to the daytime relative to the nighttime. For the patients with the most advanced stage of $\mathrm{AD}$, the estimated time of peak activity shifted from 04:00 during RLE to 11:40 after BLE. A similar protocol was used two years later, and the study was expanded to include older adults without dementia but who had sleep complaints. Similar results were obtained. Older adults with and without AD slept significantly better after two-hour exposure to 30 lux of blue light at the cornea than after exposure to the same 30 lux of red light at the cornea [48].

Researchers at the LRC also conducted a small study to verify the acceptance of the lighting solution that provides visual information as well as vertical and horizontal perceptual cues [48]. In four bedrooms in a long-term care facility in Clifton Park, NY, researchers mounted linear arrays of amber-colored LEDs to the underside of the bed frame, around the adjacent bathroom door frame, and under the mirror and handrail in the bathroom (Figs. 1 and 2). The LED array under the bed provided general, low-level ambient light at night; illuminance levels between 10 and 15 lux were measured on the floor next to the bed. The LED array fram-

a

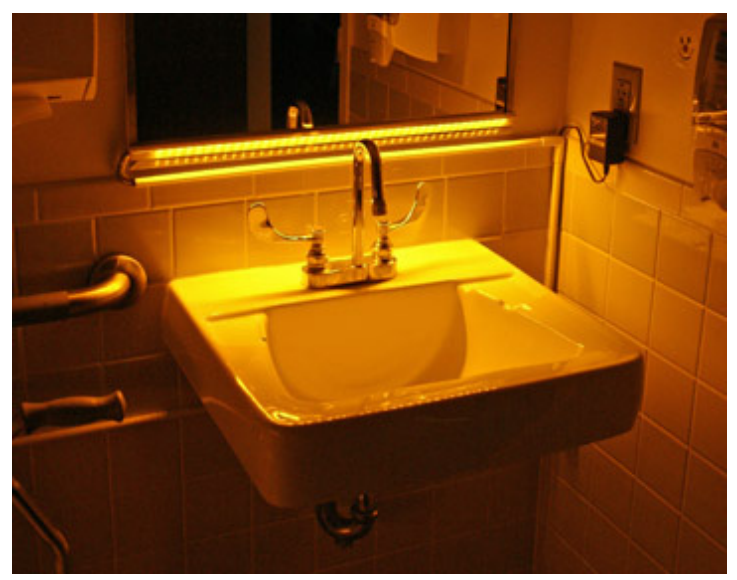

b

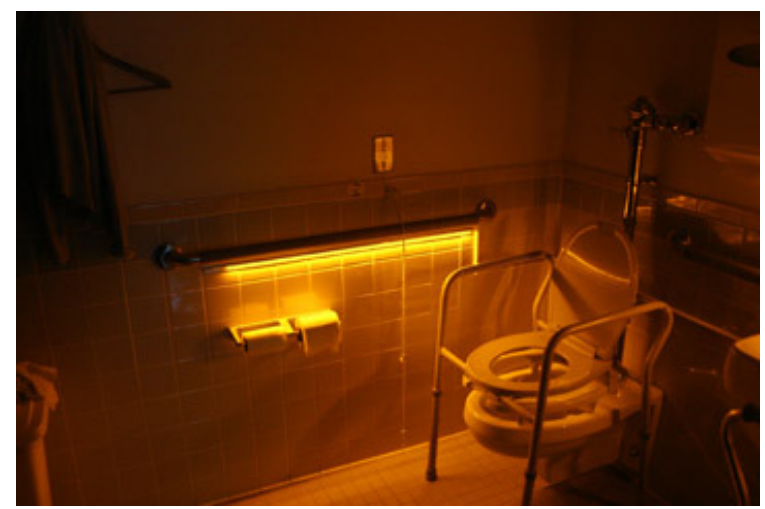

Fig. (2). a). Motion-sensor controlled amber LEDs installed at sink. b). Motion-sensor controlled amber LEDs installed at toilet. ing the bathroom door contributed approximately 2 to 10 lux on the floor near the door and when standing at the door frame, 10.5 lux was measured at the plane of the cornea. The array of LEDs in the bathroom provided 5 to 10 lux at the center of the bathroom floor and about 2 to 4 lux at the cornea when standing at the sink. Amber lights were selected because they are more efficient and less expensive than white LEDs, emit enough light to see, and are still relatively close in color to the very familiar incandescent light source. Each system was controlled by a photosensor to ensure that the LED lighting did not come on during the day or when the overhead lights were on. A motion sensor slowly turned the lights on when the residents put their feet on the floor and when nurses walked into the room. The new lighting minimized waking the residents while allowing the nursing staff to perform their mandatory nighttime bed checks. If the residents were awakened by the nurse, however, they no longer had to experience discomfort from bright overhead lights.

Before-and-after surveys of residents and staff were used to evaluate the bedroom and bathroom lighting. The afterintervention survey was conducted two weeks following the installation. Seventeen night staff members completed surveys about the pre-existing lighting conditions. Sixteen staff members completed surveys about the newly installed lighting. Twelve residents were interviewed about the preexisting lighting. The four residents who participated in the study completed surveys about the lighting installed in their bedrooms after the lighting intervention. Table 1 shows the results of the survey regarding the bedroom and bathroom lighting.

The results of the survey showed highly positive responses from both the staff and the residents to the novel nightlighting, except for the responses of the staff to the bathroom lighting who suggested that the bathroom lighting was too dim. Indeed, in confirmation of these responses, the bathroom did appear too dim to the experimenters as well. Nevertheless, the novel nightlighting was well accepted by both staff and residents in the long-term care facility. Most of the dissatisfaction was related to the amount of light available for staff to perform their tasks. In the next installation, it is proposed that either the amount of light be increased or a task light be provided to the staff to perform their rounds. Finally, Figueiro and colleagues [5] showed that the novel nightlighting system that provides horizontal and vertical perceptual cues has potential to impact postural stability and control in older adults. In a laboratory setting, Figueiro and colleagues were able to demonstrate that nightlights that provide perceptual cues can significantly reduce sway velocity in the critical, early phase of a sit-to-stand test.

\section{A Proposed 24-hr Lighting Scheme}

Based on the different studies investigating the impact of light on the visual, circadian and perceptual systems of older adults, researchers at the LRC proposed a 24-hr lighting scheme that meets older adults needs [4]. The proposed lighting scheme was designed to provide 1) high circadian stimulation during the day and low circadian stimulation at night, 2) good visual conditions during waking hours, and 3) nightlights that provide perceptual cues to increase lighting for nighttime navigation as well as increased postural stability and control while transitioning from a sitting to a stand- 
Table 1. Survey results. Sixteen staff members completed surveys about the newly installed lighting. Twelve residents were interviewed about the pre-existing lighting. The four residents who participated in the study completed surveys about the lighting installed in their bedrooms after the lighting intervention

\begin{tabular}{|c|c|c|c|}
\hline Before Installation Surveys - Staff & Yes & No & Not available \\
\hline Do you turn on the room lights for performing checks on the residents at night? & $100 \%$ & $0 \%$ & $0 \%$ \\
\hline Do the residents find the room lights uncomfortably bright at night? & $82 \%$ & $0 \%$ & $18 \%$ \\
\hline Does the staff turning on the lights at night wake you up? & $83 \%$ & $0 \%$ & $17 \%$ \\
\hline Do you find the overhead lighting bright or glaring at night? & $91 \%$ & $0 \%$ & $9 \%$ \\
\hline Is it difficult to find or reach the lighting controls at night? & $83 \%$ & $8 \%$ & $9 \%$ \\
\hline After Installation Surveys - Staff & Yes & No & Not available \\
\hline There is enough light in the bathroom without having to turn on overhead lighting. & $62 \%$ & $38 \%$ & $0 \%$ \\
\hline It is convenient to have the lighting on a motion sensor. & $100 \%$ & $0 \%$ & $0 \%$ \\
\hline After Installation Surveys - Residents & Yes & No & Not available \\
\hline Does the colored light wake you up at night when the nurses come in? & $0 \%$ & $100 \%$ & $0 \%$ \\
\hline Is the colored light uncomfortable at night if you get up to go to the bathroom? & $0 \%$ & $100 \%$ & $0 \%$ \\
\hline Do you like the colored light? & $100 \%$ & $0 \%$ & $0 \%$ \\
\hline
\end{tabular}

ing position. High circadian stimulation (CS) by light can be achieved by providing at least $400 \mathrm{~lx}$ at the cornea of a circadian effective white light source (i.e., more shortwavelength energy) during the daytime. This white light source can be daylight or any high correlated color temperature (CCT) light source, such as a $6500 \mathrm{~K}$ lamp. The use of higher CCT lamps is recommended if available in the market.

Light levels may need to be increased if the duration of exposure is shorter (i.e., two hours), but this dose/temporal relationship is yet to be established in real life applications, so the light levels recommended here are high enough and long enough to assure an effect on the circadian system of older adults. The recommended dose also takes into account the normal changes to the aging eye. No more than 100 lux at the cornea of a circadian-ineffective white light source (i.e., less short wavelength energy), such as a $2700 \mathrm{~K}$ lamp, is recommended for evening hours. The light levels were selected on the basis of estimated melatonin suppression as a function of CS after one hour exposure [12, 50]. It should be noted that the relationship between melatonin suppression and consolidation of rest/activity rhythms remains unclear. It must be true, however, that the proposed lighting scheme will provide a better light and dark pattern for the circadian system than the dim unvarying ones commonly found in senior care facilities. Moreover, the proposed lighting solution will provide older adults with a high contrast between daytime and nighttime light stimulation. When taking into account the light levels and the spectra of the light sources used during the day and at night, contrast between day and night circadian light can be as much as 15:1, while the visual light contrast is $4: 1$. The proposed lighting solution should also be glare free and shadow free, and therefore, it should satisfy the needs of the visual system of older adults. Figs. (3 to 7) show examples of how to implement the proposed lighting solutions. If addition of new lighting systems in the space is not possible (e.g., retrofit application or energy codes), the designer should consider the use of supplemental

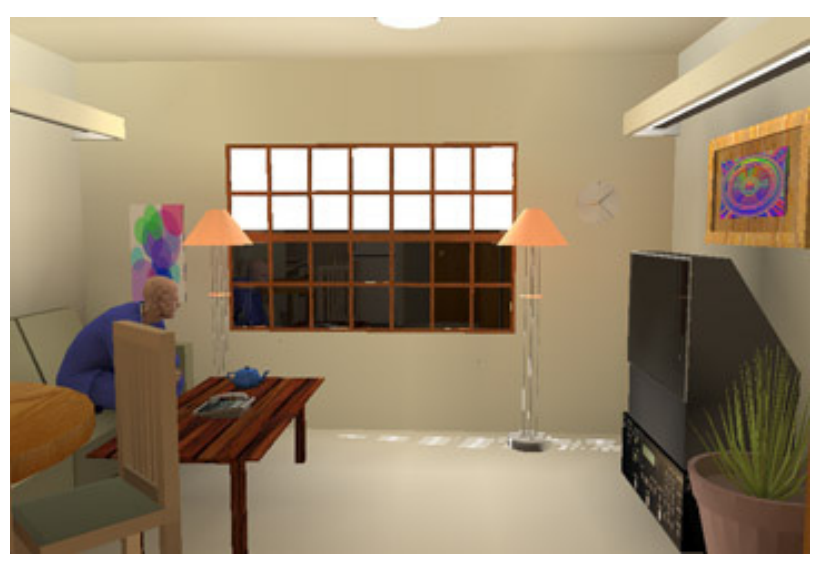

Fig. (3). Daytime view (use of high CCT lamps). 
lighting using blue LED luminaires or light boxes (peak at $470 \mathrm{~nm}$ ) providing at least $30 \mathrm{lux}$ at the cornea. This supplemental lighting can be placed on dining tables, television screens, or wheelchairs during morning hours. Finally, the designer should plan on a nightlighting system that provides sufficient illumination for the older adults to navigate in the space without disrupting their sleep as well as perceptual cues to help with balance. It is recommended that a nightlighting system such as the one described above be adopted in senior care facilities.

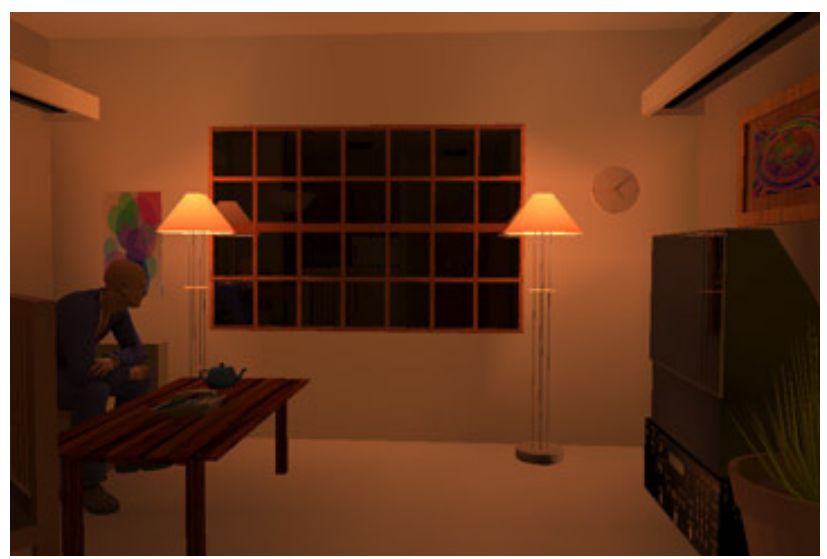

Fig. (4). Nighttime view (use of low CCT lamps).

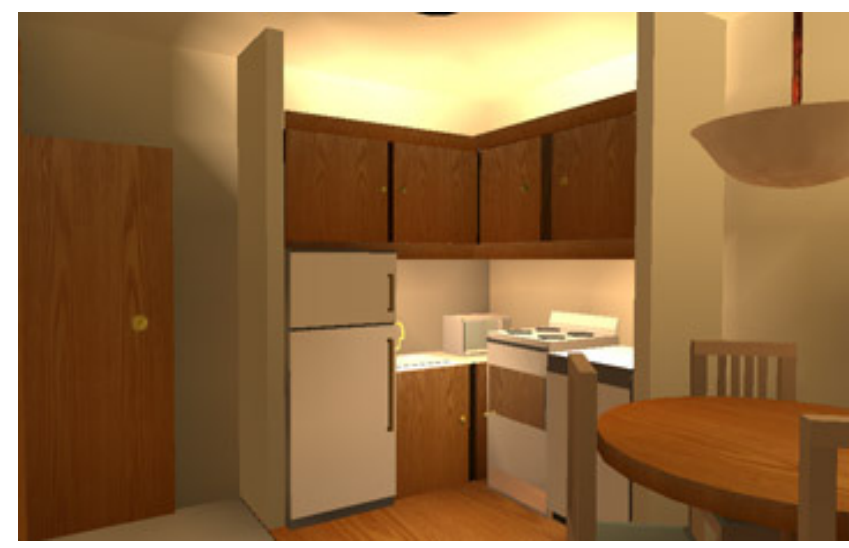

Fig. (5). View of the kitchen (low CCT lamps).

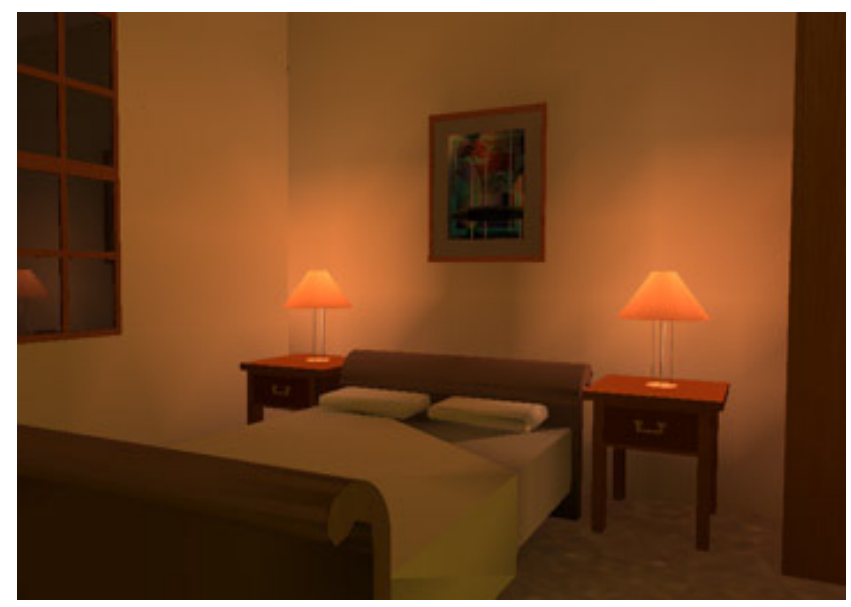

Fig. (6). View of bedroom at night (low CCT lamps).

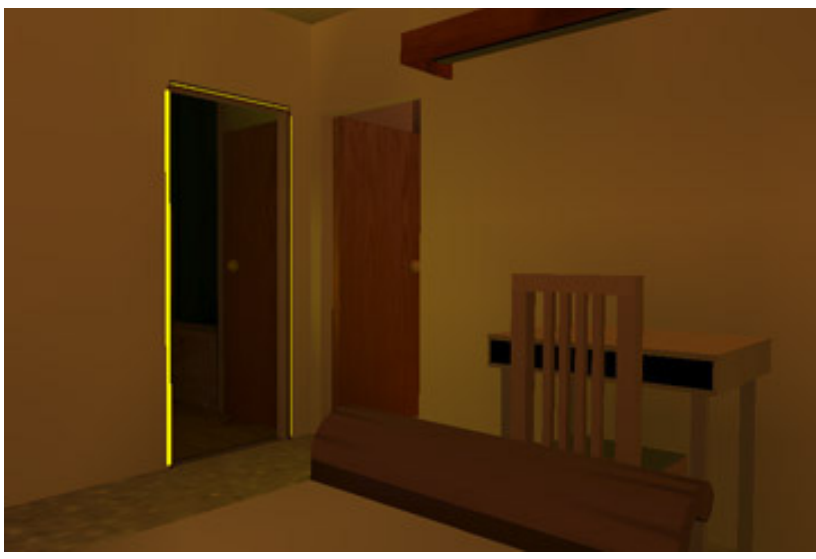

Fig. (7). View of nightlighting system.

\section{MATERIALS AND METHODOLOGY}

\section{The Demonstration Project}

The research project presented here was conducted to test the effectiveness of the proposed 24-hr lighting scheme on sleep quality of older adults. The study was approved by Rensselaer's Institute Review Board (IRB). The focus of the demonstration project was to evaluate how the use of lighting with high circadian stimulation during the day and low circadian stimulation at night would impact subjects' sleep quality. The secondary goal was to probe the subjects' overall acceptance of the new lighting in an assisted living residential setting.

\section{Location}

The demonstration project was conducted at The Terrace at Eddy Memorial, an assisted living facility in Troy, NY. The Terrace is a multi-level care residence, where most of the residents live independently but can have assistance with bathing, dressing and medication management. The rooms were private studio apartments with kitchenettes and residents spent part of their days in their rooms watching television, napping or reading books.

\section{Subjects}

Subjects were recruited by the facility's director of resident services. She identified subjects with sleep problems and asked them if they were willing to participate in a lighting study. None of the subjects were informed about the goals of the study; they were simply told that it was a lighting study. Ten female subjects were recruited for the experiment, but one subject decided to withdraw from the experiment prior to baseline data collection, one subject did not want to add the new light fixtures to in her room, and one subject had to be hospitalized during the study. A total of seven subjects completed the study. Out of the seven subjects who completed the study, one subject was legally blind (which reduces the effectiveness of the light stimulus), one subject did not stay in the room often because her husband was staying at the adjacent nursing home and she would spend most of the day with him, and one subject continuously unplugged the lamps from the wall. Because of that, we also looked at the results for the four subjects who completed the experiment without restrictions. Subjects' age ranged from 80 to 98 years old. Except for one subject, all 
had sleep complaints and three subjects were on sleep medication. Other than the subject who was legally blind, no other subjects reported any eye disease, other than normal presbyopia and reduction in contrast sensitivity due to age.

\section{Lighting Conditions}

The rooms were all illuminated with plug in table and/or floor lamps and there were no ceiling lights in the room, except for the entrance of the room (by the kitchenette) and the bathroom. All of the table and floor lamps were outfitted with incandescent lamps ranging from 60 to 150 watts (W). Light levels in the rooms were measured during the daytime using a commercial light meter. The measured daytime light levels (including daylight) in the living rooms before the lighting intervention ranged from 13 to 300 lux at plane of the cornea.

The new lighting system had to be selected to comply with the installation requirements of the facility, that is, the luminaires had to be plug-ins. The new lighting was selected to increase circadian stimulation during daylight hours. This was achieved in two ways: 1) by increasing overall daytime light levels by at least two to four times the existing light levels by adding three to four table and floor lamps in each room and 2) by using a $6500 \mathrm{~K}$ lamp, which has more shortwavelength content and was estimated to have twice the circadian stimulation as an incandescent lamp [12]. Their existing low-level incandescent table lamps were used during the evening hours. The measured daytime light levels (including daylight) in the living rooms after the lighting intervention ranged from 200 to 475 lux at the cornea. Combined, the cooler light source and the higher light levels gave a ratio between daytime and nighttime circadian stimulation of at least 4:1 and in some cases we were able to achieve a $6: 1$ ratio. The floor and table lamps were donated by Hunter Lighting (three models were used: Greenwich table lamp, Greenwich swing arm lamp, and Greenwich club floor lamp). The screwbase compact fluorescent lamps were donated by OSRAM SYLVANIA (CF23EL/MINI, 6500K). The new table and floor lamps were used with a SYLVANIA lamp and appliance indoor timer. The timer was set to turn on the lights as soon as residents woke up (it varied among subjects, but all lights were turned on between 06:45 and 08:00 hr). All the lights were programmed to be turned off at 18:00 hr. One subject "played" with the timers and her lamps were continuously found off when experimenters were visiting the facility to check on residents. One week after the new lighting was installed, she asked to change the timer so that lights were off at 16:00 hr instead of 18:00 hr. Even after the timer change, the lights were found off during period visits by the experimenters.

\section{Dependent Measures}

Two outcome measures were used to assess sleep quality before and after the lighting intervention. The first outcome measure was the Pittsburgh Sleep Quality Index (PSQI), which is widely accepted as a useful instrument for measuring sleep quality in various groups of patients. The questionnaire can be filled out in 5-10 minutes and has been shown to have good validity for patients with psychiatric and sleep disorders. The PSQI contains 19-item self-report questions that yield seven component scores: subjective sleep quality, sleep latency, duration, habitual sleep efficiency, sleep dis- turbances, use of sleep medication, and daytime dysfunction. A Global PSQI score of 5 or higher has been associated with sleep disturbance. The second outcome measure was the use of the actigraph, which is a small wrist activity monitor that is widely accepted as useful instrument for measuring rest/activity rhythms. The actigraph is used to obtain objective assessment of sleep quality in the field and has been shown to correlate well with more traditional measures of sleep quality, such as polysomnography [51]. A questionnaire probing their subjective assessment of the lighting in their room before and after the intervention was also obtained.

\section{Procedures}

The experiment ran from August 2007 to November 2007. Baseline data were collected between August 13 and August 27, 2007. Upon arrival in their rooms, researchers interviewed the subjects and asked them questions from the PSQI and the lighting assessment questionnaire. Once the interview was complete (about 15 minutes), subjects were given the actigraphs and asked to wear them all the times except when they were bathing or showering. Researchers also performed light measurements in each room to document baseline lighting conditions. Researchers stopped by the facility every other day to check on the subjects, but none of them had problems wearing the actigraphs and, in general, they kept them on at all times. The actigraphs were worn for 2 weeks, after which researchers went back to the facility to collect them and download the baseline data. During the weeks of September 12 and September 24, the new lighting was installed in each of the rooms. All the rooms had their new lights controlled by timers to assure that lights were turned on and off at specific times during the experiment. The lights were kept on for 4 weeks (from September 24 to October 22). During the last two weeks of the lighting intervention period (between October 8 and October 22), subjects were asked to wear the actigraphs once again. Between October 22 and October 24, researchers went back to the facility to collect the actigraphs and perform another interview to assess their sleep quality (PSQI) and probe their acceptance of the new lighting. At that time, the new lighting was turned off and researchers came back a week later to put the actigraphs on for another 2 weeks. Ideally, a period of at least 2 weeks should have been used to allow their circadian systems to go back to baseline, but due to the deadline of the project, this was not possible. Researchers performed a second baseline data collection to check whether their sleep quality would go back to the baseline values obtained prior to the lighting intervention.

\section{RESULTS}

Two analyses were performed on the data. The first analysis (analysis 1) included data from all seven subjects who completed the experiment, regardless of the problems during the study that may have led to less light exposure during the day. The second analysis (analysis 2) included only the subjects who, through observations made by the researchers, were perceived to be in their rooms more often during the intervention period, and therefore, were more likely to have received more circadian light during the day. We also compared the two sets of baseline data (the one collected in August and the one collected in November). Although the levels collected during the second baseline data 
were not exactly the same as the levels during baseline 1, there was no statistical significant difference between the dependent measures for baseline 1 and 2 therefore, baseline 2 was not used in the analyses.

\section{Data Analyses}

The 19 self-rated items of the PSQI questionnaire were combined to form seven component scores, each of which has a range of 0-3 points. In all cases, a score of " 0 " indicates no difficulty, while a score of " 3 " indicates severe difficulty. The seven component scores are added to give the Global PSQI score that ranges from 0-21 points, with " 0 " indicating no difficulty and " 21 " indication severe difficulty in all areas. As mentioned above, a PSQI of 5 or above indicates sleep problems. As an indication of consolidation of circadian rest/activity rhythms [45], the following measures were calculated from the actigraph data: 1) inter-daily stability (IS), a ratio indicating the strength of coupling between the light/dark cycle and rest-activity rhythm over a 24-hour period (an increase in IS suggests an improvement); 2) intradaily variability (IV), an indication of the fragmentation of the rest/activity rhythm; i.e., the frequency of the transitions between rest and activity (a decrease in IV suggests improvement); 3) amplitude of the rest-activity rhythm, calculated as the difference between the means of the most active 10-hour period (M10) and the least active 5-hr period (L5) in the 24-hr pattern (an increase in amplitude suggests improvement); 4) light/dark ratio, calculated from the quotient of the average activity in the light period divided by the average activity in the dark period. A high light/dark ratio suggests that subjects are more awake and active during the day and more asleep and inactive at night; and 5) sleep efficiency, which is an index of the amount of time in bed that is actually spent sleeping and is calculated by dividing the actual sleep time by the time in bed and multiplying it by 100 . Finally, we compared the average rating given for each question in the lighting assessment questionnaire. One tail paired Student's t-tests were used to determine whether there were significant differences between the measurements obtained before and after the lighting intervention.

\section{PSQI}

In analysis 1 , there was no significant difference between PSQI scores given after lighting intervention and baseline 1. The average score for all the seven subjects who completed the experiment was 6.35 before and after the lighting intervention. The average score of the four subjects in analysis 2 showed a slight, but not statistically significant reduction in PSQI scores (from 6.75 to 6). It is important to note, however, that the greatest improvements in PSQI score were with subjects who had more significant sleep problems, as shown by their high score at baseline. One subject had a score of 12 before the intervention and her score went down to 7 after the lighting intervention and the other subject had a score of 11 before the intervention and her score went down to 9 after the intervention. Nevertheless, on average, there was no statistical significant difference between the Global PSQI score given before and after the lighting intervention.

\section{Actigraph}

In analysis 1, there was no statistical significant difference between IS, IV and amplitude values obtained before and after the lighting intervention Although the light/dark ratio was not statistically significantly higher after the lighting intervention in analysis 1 , it did show a statistical significant increase $(\mathrm{p}=0.04)$ in analysis 2 (Fig 8 and 9). This suggests that the four subjects who had exposure to the light were spending more time awake during the daytime hours and more time asleep during the nighttime hours. Sleep efficiency increased after light intervention in both analyses, but did not reach statistical significant difference.

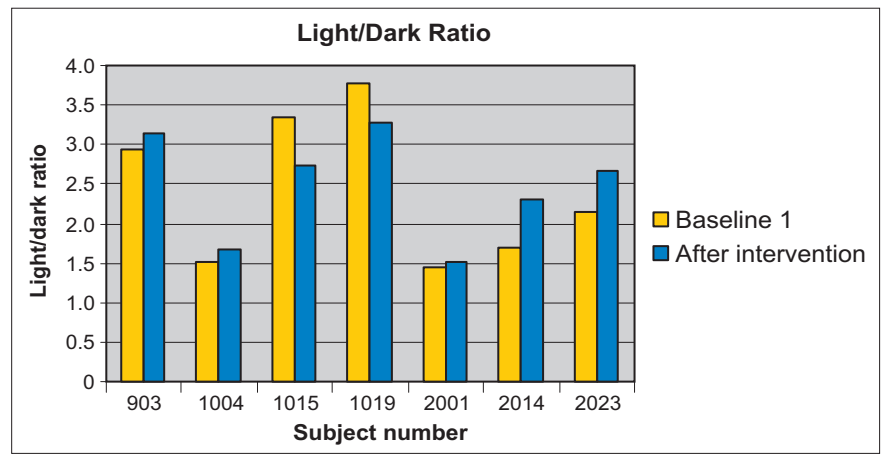

Fig. (8). Light/dark ratio for analysis 1 (seven subjects) before and after light exposure.

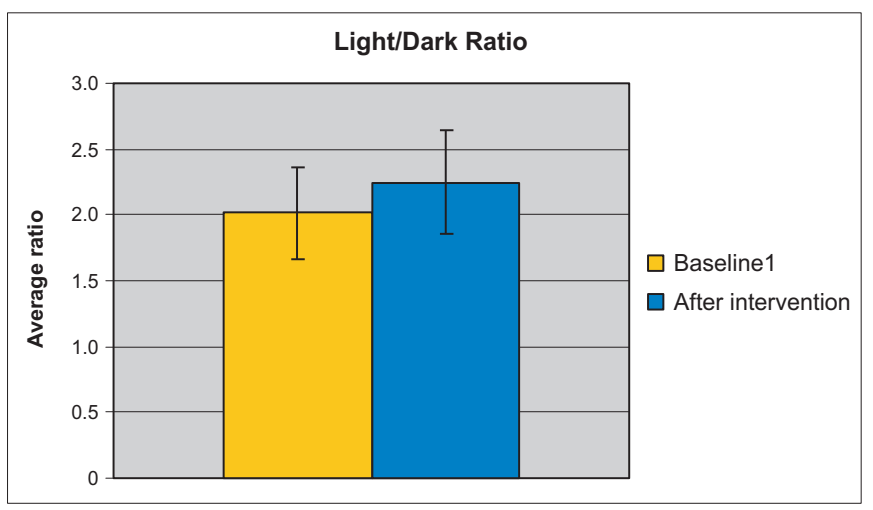

Fig. (9). Light/dark ratio for analysis 2 (four subjects) before and after intervention.

\section{Lighting Assessment}

The lighting assessment survey had questions probing daytime and nighttime lighting. Because no intervention was made in their nighttime lighting, these questions served as calibration for the subjective scale, i.e., it was expected that no significant difference between the responses would be obtained. In fact, there was no significant difference in ratings given to the nighttime lighting questions. As shown in Figs. (10 and 11), residents overwhelmingly preferred the new lighting over the existing lighting. A statistically significant higher rating $(\mathrm{p}=0.008)$ was given to the new lighting compared to their existing lighting. Moreover, subjects agreed they could read well under the new lighting and did not find the new lighting too bright. It is interesting to point out that the only subject who found the new lighting too bright was the one who "played" with the lights during the study and turned them off continuously throughout the study.

\section{DISCUSSION}

The lighting demonstration project presented here was designed to passively deliver high circadian light stimulation 


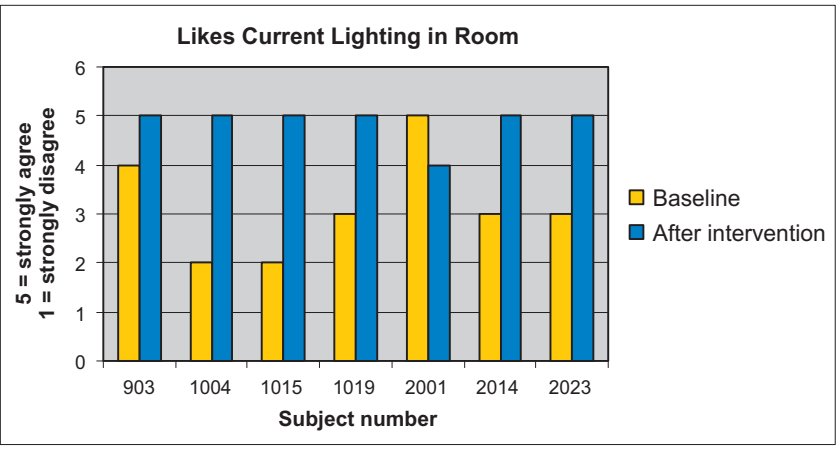

Fig. (10). Ratings given by each subject to the question: "I like the current lighting in my room."

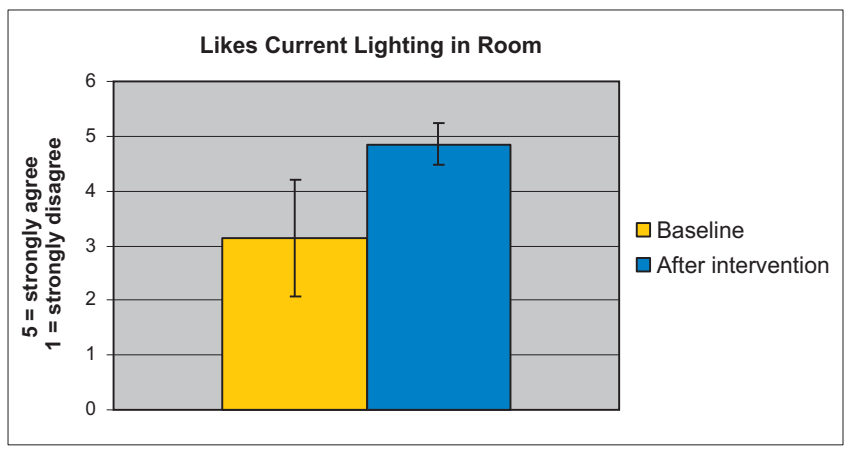

Fig. (11). Average rating given to the question: "I like the current lighting in my room."

during the day and low circadian light stimulation in the evening to older adults, while always maintaining good visibility, so as to improve both their objective and subjective measures of sleep. Seven subjects between the ages of 80 and 98 living in an assisted living facility in upstate New York were recruited for the demonstration project. The data from four of the seven subjects could be analyzed because they were consistently present during the 4-week lighting intervention. The other three subjects were, unfortunately, occupied with personal and family crises during the intervention project and their data could not be unambiguously evaluated. All four of the subjects who could be studied showed that the lighting demonstration had some positive benefits for sleep as measured through subjective reports and through wrist-actigraphic analysis. Moreover all subjects, including the subjects with personal and family crises, preferred the lighting intervention over their present lighting scheme. The present results are consistent with the results from previous studies and with theoretical expectations.

Figueiro et al. [47, 48], van Someren et al. [45], Sloane et al. [52] and Riemersma-van der Lek [53], demonstrated that light of the right spectrum and of sufficient amount applied at the proper circadian time can positively impact sleep efficiency and sleep consolidation in older adults, including those with $\mathrm{AD}$. The present results are also consistent with the knowledge that the circadian system is maximally sensitive to short wavelength radiation (blue light) and minimally sensitive to long wavelength radiation (yellow and red). In principle, when high level blue-white light (at least 400 lux at the plane of the cornea) is applied during the day and dim level yellow-white light (less than 100 lux at the plane of the cornea) is applied during the evening, the circadian system has a sufficient contrast in daily light levels to entrain to a 24-h period. The entrainment to a consistent light-dark pattern discourages random naps and intermittent sleep and supports consolidated sleep at night and activity and attention during the day, as revealed by the higher light-dark ratio observed after the light exposure.

The present findings are important for architectural practice. It is essential that theoretical research be demonstrated in the field and thereby transferred to architectural practice. There have been too few studies looking at the impact of architectural lighting on sleep quality and rest/activity rhythms of older adults in a real-life setting. The fact is that field studies are inherently difficult to conduct because (a) sites with cooperative administrators and subjects are hard to find, (b) subjects drop out of the study, and (c) funding for field demonstrations of scientific concepts is limited. With regard to the problems of the type investigated here, van Someren and colleagues were the first to demonstrate that lighting could be a non-pharmacological treatment to improve rest/activity disturbances in patients with $\mathrm{AD}$ [45]. At that time, less was known about the spectral and absolute sensitivities of the circadian system, and the light levels used in their study were probably unnecessarily high. Today, due to tuning the spectrum to the maximum sensitivity of the circadian system, light levels can be reduced and still have the same impact on rest/activity rhythms. The results of this study build upon those from van Someren [45] and Figueiro $[47,48]$ and further reinforce the call to improve lighting in senior housing based upon scientific principles.

\section{CONCLUSIONS}

This paper discusses a proposed 24-hr lighting scheme for older adults that can positively impact the aging visual, circadian and perceptual systems [4]. The new 24-hr lighting scheme discussed here appears to have important practical implications for improving the quality of life for seniors and will hopefully be adopted by architects, lighting specifiers and engineers. The authors understand that architects and designers will face challenges to implement the proposed 24$\mathrm{h}$ lighting solution due to initial costs and energy codes and regulations. These challenges should not, however, stop architects and designers from building public awareness and lobbying code officials to recognize how important lighting can be to improve the quality of life for seniors who run high risk of falls and poor sleep quality. These efforts will ultimately promote the changes we need to facilitate the adoption of superior lighting solutions in senior facilities. Moreover, the proposed lighting scheme is intended to replace inefficient incandescent light sources with more efficient light sources. The careful tuning of the spectrum of the light to the range where the circadian system is maximally sensitive (blue light) and the use of lighting controls to apply and remove the light at the right timing will reduce the need for higher energy consumption. Finally, it must be emphasized again that demonstrations of integrated, yet quantitative, lighting solutions based upon basic principles of circadian entrainment, vision and perception are essential for transforming architectural practice to serve society.

\section{ACKNOWLEDGEMENTS}

The authors would like to acknowledge the American Institute of Architects for funding the research project pre- 
sented here. The authors would also like to acknowledge the subjects, Kim Shepee-LaBombard, The Terrace staff, Patricia Rizzo, Rui Qi, Chris Munson, Howard Ohlhous, Conan O'Rourke, Xiang Wei, Dan Wang, Martin Overington, Dennis Guyon, Sandhya Parameswaran, and Russ Leslie of the Lighting Research Center for their contributions to this project. The authors would like to thank Todd Langner from Hunter Lighting for his personal support to this project and for donating all the floor and table lamps and Pamela Horner from OSRAM SYLVANIA for donating the light bulbs. EJ Van Someren is also acknowledged for his assistance with actigraphy data analyses.

\section{REFERENCES}

[1] Rea M. IESNA Lighting Handbook: Reference and Application. 9 th $^{\text {ed. }}$. New York, NY: Illuminating Engineering Society of North America; 2000.

[2] Moore R. Organization of the mammalian system. England: John Wiley and Sons; 1995.

[3] Gibson J. The senses considered as perceptual systems. Boston: Houghton Mifflin Company; 1966.

[4] Figueiro MG. A proposed 24 hour lighting scheme for older adults. Light Res Technol 2008; 40(2): 153-60.

[5] Figueiro MG, Gras L, Qi N, Rizzo P, Rea M, Rea MS. A novel lighting system for postural control and stability in seniors. Light Res. Technol 2008; 40(2): 111-26.

[6] CIE. The basis of physical photometry: Commission Internationale de l'Eclairage; 1983.

[7] CIE. Spectral luminous efficiency function for photopic vision: Commission Internationale de l'Eclairage; 1990.

[8] Moore R. Circadian Rhythms: basic neurobiology and clinical applications. Annu Rev Med 1997; 48: 253-66.

[9] Klein D, Moore R, Reppert S. Suprachiasmatic nucleus: The mind's clock. New York, NY: Oxford University Press; 1991.

[10] Berson D, Dunn F, Takao M. Phototransduction by retinal ganglion cells that set the circadian clock. Science 2002; 295(5557): 1070-3.

[11] Hattar S, Lucas RJ, Mrosovsky N, et al. Melanopsin and rod-cone photoreceptive systems account for all major accessory visual functions in mice. Nature 2003; 424: 75-81.

[12] Rea MS, Figueiro MG, Bullough JD, Bierman A. A model of phototransduction by the human circadian system. Brain Res Rev 2005; 50(2): 213-28.

[13] Thapan K, Arendt J, Skene DJ. An action spectrum for melatonin suppression: evidence for a novel non-rod, non-cone photoreceptor system in humans. J Physiol 2001; 535: 261-7.

[14] Brainard GC, Hanifin JP, Greeson JM, et al. Action spectrum for melatonin regulation in humans: evidence for a novel circadian photoreceptor. J Neurosci 2001; 21(16): 6405-12.

[15] Rea MS, Figueiro MG, Bullough JD. Circadian photobiology: An emerging framework for lighting practice and research. Light Res Technol 2002; 34(3): 177-90.

[16] Jewett M, Rimmer D, Duffy J, Klerman E, Kronauer R, Czeisler C. Human circadian pacemaker is sensitive to light throughout subjective day without evidence of transients. Am J Physiol 1997; 273: R1800-R09.

[17] McIntyre I, Norman T, Burrows G, Armstrong S. Human melatonin suppression by light is intensity dependent. J Pineal Res 1989; 6(2): 149-56.

[18] Hebert M, Martin S, Lee C, Eastman C. The effects of prior light history on the suppression of melatonin by light in humans. J Pineal Res 2002; 33(4): 198-03.

[19] Previc F. The neuropsychology of 3-D space. Psychol Bull 1998; 124(2): 123-64.

[20] Weale R. The Ageing Eye. London: HK Lewis and Company; 1963.

[21] Figueiro MG. Lighting the Way: A Key to Independence. Troy, NY: Rensselaer Polytechnic Institute; 2001.

[22] Van Someren EJ. Circadian and sleep disturbances in the elderly. Exp Gerontol 2000; 35: 1229-37.

[23] Van Cauter E, Plat L, Leproult R, Copinschi G. Alterations of circadian rhythmicity and sleep in aging: endocrine consequences. Horm Res 1998; 49: 147-52.
[24] Swaab D, Fliers E, Partiman T. The suprachiasmatic nucleus of the human brain in relation to sex, age and senile dementia. Brain Res 1985; 342(1): 37-44

[25] Hofman MA, Swaab DF. Living by the clock: the circadian pacemaker in older people. Ageing Res Rev 2006; 5(1): 33-51.

[26] Skene DJ, Swaab DF. Melatonin rhythmicity: effect of age and Alzheimer's disease. Exp Gerontol 2003; 38(1-2): 199-206.

[27] Espiritu RC, Kripke DF, Ancoli-Israel S, et al. Low illumination experienced by San Diego adults: Association with atypical depressive symptoms. Soc Biol Psychiatry 1994; 35: 403-07.

[28] Sanchez R, Ge Y, Zee P. A comparison of the strength of external zeitgeber in young and older adults. Sleep Res 1993; 22: 416.

[29] Ancoli-Israel S, Parker L, Sinaee R, Fell RL, Kripke DF. Sleep fragmentation in patients from a nursing home. J Gerontol 1989; 44(1): M18-21.

[30] Karasek M. Melatonin, human aging, and age-related diseases. Exp Gerontol 2004; 39(11-12): 1723-9.

[31] Van Someren EJ. Circadian rhythms and sleep in human aging. Chronobiol Int 2000; 17(3): 233-43.

[32] Duffy JF, Dijk DJ, Klerman EB, Czeisler CA. Later endogenous circadian temperature nadir relative to an earlier wake time in older people. Am J Physiol 1998; 275: R1478-87.

[33] Nadler M, Miller D, Nadler DJ. Glare and contrast sensitivity for clinicians. New York: Springer Verlag; 1990.

[34] De Boer MR, Lips P, Moll AC, Volker-Dieben HJ, Deeg DJ, Van Rens GH. Different aspects of visual impairment as risk factors for falls and fractures in older men and women. J Bone Miner Res 2004; 19(9): 1539-47.

[35] Fetveit A, Skjerve A, Bjorvatn B. Bright light treatment improves sleep in institutionalized elderly--an open trial. Int J Geriatr Psychiatry $2003 ; 18(6): 520-6$.

[36] Alessi C, Martin J, Webber A, Cynthia Kim E, Harker J, Josephson $\mathrm{K}$. Randomized, controlled trial of a nonpharmacological intervention to improve abnormal sleep/wake patterns in nursing home residents. Am Geriatr Soc 2005; 53(5): 803-10.

[37] Murphy P, Campbell S. Enhanced performance in elderly subjects following bright light treatment of sleep maintenance in insomnia. J Sleep Res 1996; 5: 165-72.

[38] Fontana Gasio P, Kräuchi K, Cajochen C, et al. Dawn-dusk simulation light therapy of disturbed circadian rest-activity cycles in demented elderly. Exp Gerontol 2003; 38: 207-16.

[39] Ancoli-Israel S, Martin J, Shochat T, Marler M. Morning light delays activity acrophase in demented elderly. Soc. Light Treat Biol Rhythms 2000; 12: 15.

[40] Koyama E, Matsubara H, Nakano T. Bright light treatment for sleep-wake disturbances in aged individuals with dementia. Psychiatry Clin Neurosci 1999; 53: 227-9.

[41] Lyketosos C, Lindell Veiel L, Baker A, Steele C. A randomized, controlled trial of bright light therapy for agitated behaviors in dementia patients residing in long-term care. Int J Geriatr Psychiatry 1999; 14: 520-25.

[42] Mishima K, Hishikawa Y, Okawa M. Randomized, dim light controlled, crossover test of morning bright light therapy for restactivity rhythm disorders in patients with vascular dementia and dementia of Alzheimer's type. Chronobiol Int 1998; 15: 647-54.

[43] Mishima K, Okawa M, Hishikawa Y, Hozumi S, Hori H, Takahashi K. Morning bright light therapy for sleep and behavior disorders in elderly patients with dementia. Acta Psychiatr Scand 1994; 87: 1-7.

[44] Lovell B, Ancoli-Israel S, Gevirtz R. Effect of bright light treatment on agitated behavior in institutionalized elderly subjects. Psychiatry Res 1995; 57: 7-12.

[45] Van Someren EJ, Kessler A, Mirmirann M, Swaab D. Indirect bright light improves circadian rest-activity rhythm disturbances in demented patients. Biol Psychiatry 1997; 41: 955-63.

[46] Satlin A, Volicer L, Ross V, Herz L, Campbell S. Bright light treatment for behavioral and sleep disturbances in patients with Alzheimer's disease. Am J Psychiatry 1992; 149: 1028-32.

[47] Figueiro MG, Eggleston G, Rea MS. Light therapy and Alzheimer's disease. Sleep Rev 2003; 4, 24.

[48] Figueiro MG, Rea MS. LEDs: Improving the sleep quality of older adults. In: Proceedings of the CIE Midterm Meeting and International Lighting Congress; Leon, Spain. May 12-21, 2005: 357-367.

[49] Brainard GC, Hanifin JP, Rollag MD, et al. Human melatonin regulation is not mediated by the three cone photopic visual system. J Clin Endocrinol Metab 2001; 86(1): 433-6. 
[50] Figueiro MG, Rea MS, Bullough JD. Circadian effectiveness of two polychromatic lights in suppressing human nocturnal melatonin. Neurosci Lett 2006; 406: 293-97.

[51] Van Someren EJ. Improving actigraphic sleep estimates in insomnia and dementia: how many nights? J Sleep Res 2007; 16(3): 26975 .

[52] Sloane PD, Williams CS, Mitchell CM, et al. High-intensity environmental light in dementia: effect on sleep and activity. J Am Geriatr Soc 2007; 55(10): 1524-33.
[53] Riemersma-van der Lek RF, Swaab DF, Twisk J, Hol EM, Hoogendijk WJ, Van Someren EJ. Effect of bright light and melatonin on cognitive and noncognitive function in elderly residents of group care facilities: A randomized controlled trial. JAMA 2008; 299: 2642-55.

Received: May 07, 2008

Revised: June 30, 2008

Accepted: July 03, 2008

(C) Figueiro et al.; Licensee Bentham Open.

This is an open access article distributed under the terms of the Creative Commons Attribution License (http://creativecommons.org/licenses/by/2.5/), which permits unrestrictive use, distribution, and reproduction in any medium, provided the original work is properly cited. 